International Mathematical Forum, Vol. 9, 2014, no. 18, 871 - 890

HIKARI Ltd, www.m-hikari.com

http://dx.doi.org/10.12988/imf.2014.4488

\title{
Large and Moderate Deviation Principles for Kernel Distribution Estimator
}

\author{
Yousri Slaoui \\ Université de Poitiers \\ Laboratoire de Mathématiques et Application \\ Futuroscope Chasseneuil, France
}

Copyright (c) 2014 Yousri Slaoui. This is an open access article distributed under the Creative Commons Attribution License, which permits unrestricted use, distribution, and reproduction in any medium, provided the original work is properly cited.

\begin{abstract}
In this paper we prove large and moderate deviations principles for the kernel estimator of a distribution function introduced by Nadaraya [1964. Some new estimates for distribution functions. Theory Probab. Appl. 9, 497500]. We provide results both for the pointwise and the uniform deviations.
\end{abstract}

Mathematics Subject Classifiation: 62E20, 60F10

Keywords: Distribution estimation; Large and Moderate deviations principles

\section{Introduction}

Let $X_{1}, \ldots, X_{n}$ be independent, identically distributed of random variables, and let $f$ and $F$ denote respectively the probability density of $X_{1}$ and the distribution function of $X_{1}$. Nadaraya (1964) introduce a kernel $K$ (that is, a function satisfying $\int_{\mathbb{R}} K(x) d x=1$ ), a function $\mathcal{K}$ (that is, a function defined by $\mathcal{K}(z)=\int_{-\infty}^{z} K(u) d u$ ), and a bandwidth $\left(h_{n}\right)$ (that is, a sequence of positive real numbers that goes to zero). The estimator proposed by Nadaraya (1964) to estimate the distribution function $F$ at the point $x$ is given by

$$
F_{n}(x)=\frac{1}{n} \sum_{k=1}^{n} \mathcal{K}\left(\frac{x-X_{k}}{h_{n}}\right) .
$$


Some theoretical properties of the estimator $F_{n}$ have been investigated (see among many others, Nadaraya (1964), Reiss (1981), and Hill (1985)). Reiss (1981) and Falk (1983) showed that the kernel distribution estimator (1) have an asymptotically better performance than empirical distribution function, which does not take into account the smoothness of $F$.

Recently, large and moderate deviations results have been proved for the wellknown nonrecursive kernel density estimator introduced by Rosenblatt (1956) (see also Parzen, 1962). The large deviations principle has been studied by Louani (1998) and Worms (2001). Gao (2003) and Mokkadem et al. (2005) extend these results and provide moderate deviations principles. The purpose of this paper is to establish large and moderate deviations principles for the nonrecursive distribution estimator (1).

Let us first recall that a $\mathbb{R}^{m}$-valued sequence $\left(Z_{n}\right)_{n \geq 1}$ satisfies a large deviations principle (LDP) with speed $\left(\nu_{n}\right)$ and good rate function $I$ if :

1. $\left(\nu_{n}\right)$ is a positive sequence such that $\lim _{n \rightarrow \infty} \nu_{n}=\infty$;

2. $I: \mathbb{R}^{m} \rightarrow[0, \infty]$ has compact level sets;

3. for every borel set $B \subset \mathbb{R}^{m}$,

$$
\begin{aligned}
-\inf _{x \in B} I(x) & \leq \liminf _{n \rightarrow \infty} \nu_{n}^{-1} \log \mathbb{P}\left[Z_{n} \in B\right] \\
& \leq \limsup _{n \rightarrow \infty} \nu_{n}^{-1} \log \mathbb{P}\left[Z_{n} \in B\right] \leq-\inf _{x \in \bar{B}} I(x),
\end{aligned}
$$

where $\stackrel{\circ}{B}$ and $\bar{B}$ denote the interior and the closure of $B$ respectively. Moreover, let $\left(v_{n}\right)$ be a nonrandom sequence that goes to infinity; if $\left(v_{n} Z_{n}\right)$ satisfies a LDP, then $\left(Z_{n}\right)$ is said to satisfy a moderate deviations principle (MDP).

The first aim of this paper is to establish pointwise LDP for the kernel distribution estimator (1).

We show that using the bandwiths defined as $h_{n}=h(n)$ for all $n$, where $h$ is a regularly varing function with exponent $(-a), a \in] 0,1[$. We prove that the sequence $\left(F_{n}(x)-F(x)\right)$ satisfies a LDP with speed $(n)$ and the rate function defined as follows:

$$
\left\{\begin{array}{l}
\text { if } F(x) \neq 0, \quad I_{x}: t \rightarrow F(x) I\left(1+\frac{t}{F(x)}\right) \\
\text { if } F(x)=0, \quad I_{x}(0)=0 \quad \text { and } I_{x}(t)=+\infty \text { for } t \neq 0
\end{array}\right.
$$

where

$$
\begin{aligned}
& I(t)=\sup _{u \in \mathbb{R}}\{u t-\psi(u)\} \\
& \psi(u)=\exp (u)-1 .
\end{aligned}
$$


Our second aim is to provide pointwise MDP for the distribution estimator defined by $(1)$. For any positive sequence $\left(v_{n}\right)$ satisfying

$$
\lim _{n \rightarrow \infty} v_{n}=\infty \quad \text { and } \quad \lim _{n \rightarrow \infty} \frac{v_{n}^{2}}{n}=0
$$

and general bandwidths $\left(h_{n}\right)$, we prove that the sequence

$$
v_{n}\left(F_{n}(x)-F(x)\right)
$$

satisfies a LDP of speed $\left(n / v_{n}^{2}\right)$ and rate function $J_{x}($.$) defined by$

$$
\begin{cases}\text { if } & f(x) \neq 0, \quad J_{x}: t \rightarrow \frac{t^{2}}{2 F(x)} \\ \text { if } \quad f(x)=0, & J_{x}(0)=0 \quad \text { and } \quad J_{x}(t)=+\infty \quad \text { for } \quad t \neq 0 .\end{cases}
$$

Finally, we give a uniform version of the previous results. More precisely, let $U$ be a subset of $\mathbb{R}$; we establish large and moderate deviations principles for the sequence $\left(\sup _{x \in U}\left|F_{n}(x)-F(x)\right|\right)$.

\section{Assumptions and main results}

We define the following class of regularly varying sequences.

Definition 1. Let $\gamma \in \mathbb{R}$ and $\left(v_{n}\right)_{n \geq 1}$ be a nonrandom positive sequence. We say that $\left(v_{n}\right) \in \mathcal{G S}(\gamma)$ if

$$
\lim _{n \rightarrow+\infty} n\left[1-\frac{v_{n-1}}{v_{n}}\right]=\gamma
$$

Condition (4) was introduced by Galambos and Seneta (1973) to define regularly varying sequences (see also Bojanic and Seneta, 1973). Typical sequences in $\mathcal{G S}(\gamma)$ are, for $b \in \mathbb{R}, n^{\gamma}(\log n)^{b}, n^{\gamma}(\log \log n)^{b}$, and so on.

\subsection{Pointwise LDP for the Nadaraya's distribution estima- tor}

To establish pointwise LDP for $F_{n}$, we need the following assumptions.

(L1) $K: \mathbb{R} \rightarrow \mathbb{R}$ is a bounded and integrable function satisfying $\int_{\mathbb{R}} K(z) d z=1$, and $\int_{\mathbb{R}} z K(z) d z=0$.

$(\mathrm{L} 2)\left(h_{n}\right) \in \mathcal{G S}(-a)$ with $\left.a \in\right] 0,1[$. 
The following Theorem gives the pointwise LDP for $F_{n}$ in this case.

Theorem 1 (Pointwise LDP for Nadaraya's distribution estimator).

Let Assumptions $(L 1)$ and $(L 2)$ hold and assume that $F$ is continuous at $x$. Then, the sequence $\left(F_{n}(x)-F(x)\right)$ satisfies a LDP with speed $(n)$ and rate function defined by (2).

\subsection{Pointwise MDP for the Nadaraya's distribution estima- tor}

Let $\left(v_{n}\right)$ be a positive sequence; we assume that

(M1) $K: \mathbb{R} \rightarrow \mathbb{R}$ is a continuous, bounded function satisfying $\int_{\mathbb{R}} K(z) d z=1$, and, $\int_{\mathbb{R}} z K(z) d z=0$ and $\int_{\mathbb{R}} z^{2}|K(z)| d z<\infty$.

$(\mathrm{M} 2)\left(h_{n}\right) \in \mathcal{G S}(-a)$ with $\left.a \in\right] 0,1[$.

(M3) $F$ is bounded, twice differentiable, and $F^{(2)}(x)$ is bounded.

(M4) $\lim _{n \rightarrow \infty} v_{n}=\infty$ and $\lim _{n \rightarrow \infty} \frac{v_{n}^{2}}{n}=0$.

The following Theorem gives the pointwise MDP for $F_{n}$.

Theorem 2 (Pointwise MDP for the kernel distribution estimator (1)).

Let Assumptions $(M 1)-(M 4)$ hold and assume that $F$ is continuous at $x$. Then, the sequence $\left(F_{n}(x)-F(x)\right)$ satisfies a MDP with speed $\left(n / v_{n}^{2}\right)$ and rate function $J_{x}$ defined in $(3)$.

\subsection{Uniform LDP and MDP for the Nadaraya's distribution estimator}

To establish uniform large deviations principles for the distribution estimator defined by (1) on a bounded set, we need the following assumptions:

(U1) i) $\int_{\mathbb{R}} z K(z) d z=0$ and $\int_{\mathbb{R}} z^{2}|K(z)| d z<\infty$.

ii) $\mathcal{K}$ is Hölder continuous.

(U2) $F$ is bounded, twice differentiable, and, $\sup _{x \in \mathbb{R}}\left|F^{(2)}(x)\right|<\infty$.

(U3) $\lim _{n \rightarrow \infty} \frac{v_{n}^{2} \log v_{n}}{n}=0$.

Set $U \subseteq \mathbb{R}$; in order to state in a compact form the uniform large and moderate deviations principles for the distribution estimator defined by (1) on $U$, we set:

$$
\begin{aligned}
& g_{U}(\delta)=\left\{\begin{array}{lll}
\|F\|_{U, \infty} I\left(1+\frac{\delta}{\|F\|_{U, \infty}}\right) & \text { when } v_{n} \equiv 1 \quad, \quad(L 1) \text { and }(L 2) \text { hold } \\
\frac{\delta^{2}}{2\|F\|_{U, \infty}} & \text { when } v_{n} \rightarrow \infty \quad, \quad(M 1)-(M 4) \text { hold }
\end{array}\right. \\
& \tilde{g}_{U}(\delta)=\min \left\{g_{U}(\delta), g_{U}(-\delta)\right\}
\end{aligned}
$$


where $\|F\|_{U, \infty}=\sup _{x \in U}|F(x)|$.

Remark 1. The functions $g_{U}($.$) and \tilde{g}_{U}($.$) are non-negative, continuous, increasing$ on $] 0,+\infty[$ and decreasing on $]-\infty, 0\left[\right.$, with a unique global minimum in $0\left(\tilde{g}_{U}(0)=\right.$ $\left.g_{U}(0)=0\right)$. They are thus good rate functions (and $g_{U}($.$) is strictly convex).$

Theorem 3 below states uniform LDP on $U$ in the case $U$ is bounded, and Theorem 4 in the case $U$ is unbounded.

Theorem 3 (Uniform deviations on a bounded set for the kernel distribution estimator (1)). Let $(U 1)-(U 3)$ hold. Then for any bounded subset $U$ of $\mathbb{R}$ and for all $\delta>0$,

$$
\lim _{n \rightarrow \infty} n^{-1} v_{n}^{2} \log \mathbb{P}\left[\sup _{x \in U} v_{n}\left|F_{n}(x)-F(x)\right| \geq \delta\right]=-\tilde{g}_{U}(\delta)
$$

To establish uniform large deviations principles for the distribution estimator (1) on an unbounded set, we need the following additionnal assumptions:

(U4) i) There exists $\beta>0$ such that $\int_{\mathbb{R}}\|x\|^{\beta} f(x) d x<\infty$.

ii) $F$ is uniformly continuous.

(U5) There exists $\tau>0$ such that $z \mapsto\|z\|^{\tau} \mathcal{K}(z)$ is a bounded function.

(U6) i) There exists $\zeta>0$ such that $\int_{\mathbb{R}}\|z\|^{\zeta}|K(z)| d z<\infty$

ii) There exists $\eta>0$ such that $z \mapsto\|z\|^{\eta} F(z)$ is a bounded function.

Theorem 4 (Uniform deviations on an unbounded set for the estimator defined by (1)). Let $(U 1)-(U 6)$ hold. Then for any subset $U$ of $\mathbb{R}$ and for all $\delta>0$,

$$
\begin{aligned}
-\tilde{g}_{U}(\delta) & \leq \liminf _{n \rightarrow \infty} n^{-1} v_{n}^{2} \log \mathbb{P}\left[\sup _{x \in U} v_{n}\left|F_{n}(x)-F(x)\right| \geq \delta\right] \\
& \leq \limsup _{n \rightarrow \infty} n^{-1} v_{n}^{2} \log \mathbb{P}\left[\sup _{x \in U} v_{n}\left|F_{n}(x)-F(x)\right| \geq \delta\right] \leq-\frac{\beta}{\beta+1} \tilde{g}_{U}(\delta)
\end{aligned}
$$

The following corollary is a straightforward consequence of Theorem 4 .

Corollary 1. Under the assumptions of Theorem 4, if $\int_{\mathbb{R}}\|x\|^{\xi} F(x) d x<\infty$ for all $\xi$ in $\mathbb{R}$, then for any subset $U$ of $\mathbb{R}$,

$$
\lim _{n \rightarrow \infty} n^{-1} v_{n}^{2} \log \mathbb{P}\left[\sup _{x \in U} v_{n}\left|F_{n}(x)-F(x)\right| \geq \delta\right]=-\tilde{g}_{U}(\delta)
$$

Comment. Since the sequence $\left(\sup _{x \in U}\left|F_{n}(x)-F(x)\right|\right)$ is positive and since $\tilde{g}_{U}$ is continuous on $[0,+\infty$ [, increasing and goes to infinity as $\delta \rightarrow \infty$, the application of Lemma 5 in Worms (2001) allows to deduce from (5) or (6) that $\sup _{x \in U}\left|F_{n}(x)-F(x)\right|$ satisfies a LDP with speed $(n)$ and good rate function $\tilde{g}_{U}$ on $\mathbb{R}+$. 


\section{Proofs}

Throught this section we use the following notation:

$$
Y_{k, n}=\mathcal{K}\left(\frac{x-X_{k}}{h_{n}}\right)
$$

Noting that, in view of (1), we have

$$
F_{n}(x)-\mathbb{E}\left[F_{n}(x)\right]=\frac{1}{n} \sum_{k=1}^{n}\left(Y_{k, n}-\mathbb{E}\left[Y_{k, n}\right]\right)
$$

Let $\left(\Psi_{n}\right)$ and $\left(B_{n}\right)$ be the sequences defined as

$$
\begin{aligned}
& \Psi_{n}(x)=\frac{1}{n} \sum_{k=1}^{n}\left(Y_{k, n}-\mathbb{E}\left[Y_{k, n}\right]\right) \\
& B_{n}(x)=\mathbb{E}\left[F_{n}(x)\right]-F(x)
\end{aligned}
$$

We have:

$$
F_{n}(x)-F(x)=\Psi_{n}(x)+B_{n}(x)
$$

Theorems 1, 2, 3 and 4 are consequences of (8) and the following propositions.

Proposition 1 (Pointwise LDP and MDP for $\left(\Psi_{n}\right)$ ).

1. Under the assumptions $(L 1)$ and $(L 2)$, the sequence $\left(F_{n}(x)-\mathbb{E}\left(F_{n}(x)\right)\right)$ satisfies a LDP with speed $(n)$ and rate function $I_{x}$.

2. Under the assumptions $(M 1)-(M 4)$, the sequence $\left(v_{n} \Psi_{n}(x)\right)$ satisfies a LDP with speed $\left(n / v_{n}^{2}\right)$ and rate function $J_{x}$.

Proposition 2 (Uniform LDP and MDP for $\left(\Psi_{n}\right)$ ).

1. Let $(U 1)-(U 3)$ hold. Then for any bounded subset $U$ of $\mathbb{R}$ and for all $\delta>0$,

$$
\lim _{n \rightarrow \infty} n^{-1} v_{n}^{2} \log \mathbb{P}\left[\sup _{x \in U} v_{n}\left|\Psi_{n}(x)\right| \geq \delta\right]=-\tilde{g}_{U}(\delta)
$$

2. Let $(U 1)-(U 6)$ hold. Then for any subset $U$ of $\mathbb{R}$ and for all $\delta>0$,

$$
\begin{aligned}
-\tilde{g}_{U}(\delta) & \leq \liminf _{n \rightarrow \infty} n^{-1} v_{n}^{2} \log \mathbb{P}\left[\sup _{x \in U} v_{n}\left|\Psi_{n}(x)\right| \geq \delta\right] \\
& \leq \limsup _{n \rightarrow \infty} n^{-1} v_{n}^{2} \log \mathbb{P}\left[\sup _{x \in U} v_{n}\left|\Psi_{n}(x)\right| \geq \delta\right] \leq-\frac{\xi}{\xi+d} \tilde{g}_{U}(\delta)
\end{aligned}
$$


Proposition 3 (Pointwise and uniform convergence rate of $\left(B_{n}\right)$ ).

Let Assumptions (M1) - (M3) hold.

1. If $f^{\prime}$ is continuous at $x$. We have

If $a \leq 1 / 3$, then

$$
B_{n}(x)=O\left(h_{n}^{2}\right)
$$

If $a>1 / 3$, then

$$
B_{n}(x)=o\left(\sqrt{n^{-1} h_{n}}\right) .
$$

2. If (U2) holds, then:

If $a \leq 1 / 3$, then

$$
\sup _{x \in \mathbb{R}}\left|B_{n}(x)\right|=O\left(h_{n}^{2}\right)
$$

If $a>1 / 3$, then

$$
\sup _{x \in \mathbb{R}}\left|B_{n}(x)\right|=o\left(\sqrt{n^{-1} h_{n}}\right) .
$$

Set $x \in \mathbb{R}$; since the assumptions of Theorems 1 guarantee that $\lim _{n \rightarrow \infty} B_{n}(x)=$ 0, Theorem 1 is a straightforward consequence of the application of Part 1 (respectively of Part 2) of Proposition 1. Moreover, under the assumptions of Theorem 2, we have by application of Propostion $3, \lim _{n \rightarrow \infty} v_{n} B_{n}(x)=0$; Theorem 2 thus straightfully follows from the application of Part 3 of Proposition 1. Finaly, Theorem 3 and 4 follows from Proposition 2 and the second part of Proposition 3.

We now state a preliminary lemma, which will be used in the proof of Proposition 1. For any $u \in \mathbb{R}$, Set

$$
\begin{aligned}
\Lambda_{n, x}(u) & =n^{-1} v_{n}^{2} \log \mathbb{E}\left[\exp \left(u \frac{n}{v_{n}} \Psi_{n}(x)\right)\right] \\
\Lambda_{x}^{L}(u) & =F(x)(\psi(u)-u), \\
\Lambda_{x}^{M}(u) & =\frac{u^{2}}{2} F(x)
\end{aligned}
$$

Lemma 1. [Convergence of $\left.\Lambda_{n, x}\right]$

1. (Pointwise convergence)

If $F$ is continuous at $x$, then for all $u \in \mathbb{R}$

$$
\lim _{n \rightarrow \infty} \Lambda_{n, x}(u)=\Lambda_{x}(u)
$$

where

$$
\Lambda_{x}(u)=\left\{\begin{array}{lll}
\Lambda_{x}^{L}(u) & \text { when } & v_{n} \equiv 1 \\
\Lambda_{x}^{M}(u) & \text { when } & v_{n} \rightarrow \infty
\end{array}\right.
$$


2. (Uniform convergence)

If $F$ is uniformly continuous, then the convergence (9) holds uniformly in $x \in U$.

Our proofs are now organized as follows: Lemma 1 is proved in Section 3.1, Proposition 1 in Section 3.4 and Proposition 2 in Section 3.3.

\subsection{Proof of Lemma 1.}

Set $u \in \mathbb{R}, u_{n}=u / v_{n}$ and $a_{n}=n$. We have:

$$
\begin{aligned}
\Lambda_{n, x}(u) & =\frac{v_{n}^{2}}{a_{n}} \log \mathbb{E}\left[\exp \left(u_{n} a_{n} \Psi_{n}(x)\right)\right] \\
& =\frac{v_{n}^{2}}{a_{n}} \log \mathbb{E}\left[\exp \left(u_{n} \sum_{k=1}^{n}\left(Y_{k, n}-\mathbb{E}\left[Y_{k, n}\right]\right)\right)\right] \\
& =\frac{v_{n}^{2}}{a_{n}} \sum_{k=1}^{n} \log \mathbb{E}\left[\exp \left(u_{n} Y_{k, n}\right)\right]-u v_{n} \mathbb{E}\left[Y_{1, n}\right]
\end{aligned}
$$

By Taylor expansion, there exists $c_{k, n}$ between 1 and $\mathbb{E}\left[\exp \left(u_{n} Y_{k, n}\right)\right]$ such that

$$
\log \mathbb{E}\left[\exp \left(u_{n} Y_{k, n}\right)\right]=\mathbb{E}\left[\exp \left(u_{n} Y_{k, n}\right)-1\right]-\frac{1}{2 c_{k, n}^{2}}\left(\mathbb{E}\left[\exp \left(u_{n} Y_{k, n}\right)-1\right]\right)^{2}
$$

and $\Lambda_{n, x}$ can be rewriten as

$$
\begin{aligned}
\Lambda_{n, x}(u)= & \frac{v_{n}^{2}}{a_{n}} \sum_{k=1}^{n} \mathbb{E}\left[\exp \left(u_{n} Y_{k, n}\right)-1\right]-\frac{v_{n}^{2}}{2 a_{n}} \sum_{k=1}^{n} \frac{1}{c_{k, n}^{2}}\left(\mathbb{E}\left[\exp \left(u_{n} Y_{k, n}\right)-1\right]\right)^{2} \\
& -u v_{n} \mathbb{E}\left[Y_{1, n}\right]
\end{aligned}
$$

First case: $v_{n} \rightarrow \infty$. A Taylor's expansion implies the existence of $c_{k, n}^{\prime}$ between 0 and $u_{n} Y_{k, n}$ such that

$$
\mathbb{E}\left[\exp \left(u_{n} Y_{k, n}\right)-1\right]=u_{n} \mathbb{E}\left[Y_{k, n}\right]+\frac{1}{2} u_{n}^{2} \mathbb{E}\left[Y_{k, n}^{2}\right]+\frac{1}{6} u_{n}^{3} \mathbb{E}\left[Y_{k, n}^{3} e^{c_{k, n}^{\prime}}\right]
$$

Therefore,

$$
\begin{aligned}
\Lambda_{n, x}(u)= & \frac{1}{2} u^{2} a_{n} \sum_{k=1}^{n} \mathbb{E}\left[Y_{k, n}^{2}\right]+\frac{1}{6} u^{2} \frac{u_{n}}{a_{n}} \sum_{k=1}^{n} \mathbb{E}\left[Y_{k, n}^{3} e^{c_{k, n}^{\prime}}\right] \\
& -\frac{v_{n}^{2}}{2 a_{n}} \sum_{k=1}^{n} \frac{1}{c_{k, n}^{2}}\left(\mathbb{E}\left[\exp \left(u_{n} Y_{k, n}\right)-1\right]\right)^{2} \\
= & \frac{1}{2} u^{2} F(x)+R_{n, x}^{(1)}(u)+R_{n, x}^{(2)}(u)
\end{aligned}
$$


with

$$
\begin{aligned}
R_{n, x}^{(1)}(u) & =u^{2} \int_{\mathbb{R}} K(z) \mathcal{K}(-z)\left[F\left(x+z h_{n}\right)-F(x)\right] d z \\
R_{n, x}^{(2)}(u) & =\frac{1}{6} \frac{u^{3}}{v_{n}} \frac{1}{a_{n}} \sum_{k=1}^{n} \mathbb{E}\left[Y_{k}^{3} e^{c_{k, n}^{\prime}}\right]-\frac{v_{n}^{2}}{2 a_{n}} \sum_{k=1}^{n} \frac{1}{c_{k, n}^{2}}\left(\mathbb{E}\left[\exp \left(u_{n} Y_{k, n}\right)-1\right]\right)^{2}
\end{aligned}
$$

Since $F$ is continuous, we have $\lim _{n \rightarrow \infty}\left|F\left(x+z h_{n}\right)-F(x)\right|=0$, and thus, by the dominated convergence theorem, (M1) implies that

$$
\lim _{n \rightarrow \infty} \int_{\mathbb{R}} K(z) \mathcal{K}(-z)\left|F\left(x+z h_{n}\right)-F(x)\right| d z=0
$$

it follows that $\lim _{n \rightarrow \infty}\left|R_{n, x}^{(1)}(u)\right|=0$.

Moreover, in view of (7), we have $\left|Y_{k, n}\right| \leq\|\mathcal{K}\|_{\infty}$, then

$$
\begin{aligned}
c_{k, n}^{\prime} & \leq\left|u_{n} Y_{k, n}\right| \\
& \leq\left|u_{n}\right|\|\mathcal{K}\|_{\infty}
\end{aligned}
$$

Noting that $\mathbb{E}\left|Y_{k, n}\right|^{3} \leq 3\|F\|_{\infty} \int_{\mathbb{R}}|K(z)|\left|\mathcal{K}^{2}(z)\right| d z$. Hence, it follows from (12), there exists a positive constant $c_{1}$ such that, for $n$ large enough,

$$
\left|\frac{u^{3}}{v_{n}} \frac{1}{a_{n}} \sum_{k=1}^{n} \mathbb{E}\left[Y_{k}^{3} e^{c_{k, n}^{\prime}}\right]\right| \leq c_{1} e^{\left|u_{n}\right|\|\mathcal{K}\|_{\infty}} \frac{u^{3}}{v_{n}}\|F\|_{\infty} \int_{\mathbb{R}}|K(z)|\left|\mathcal{K}^{2}(z)\right| d z
$$

which goes to 0 as $n \rightarrow \infty$ since $v_{n} \rightarrow \infty$.

In the same way, there exists a positive constant $c_{2}$ such that, for $n$ large enough,

$$
\begin{aligned}
& \left|\frac{v_{n}^{2}}{2 a_{n}} \sum_{k=1}^{n} \frac{1}{c_{k, n}^{2}}\left(\mathbb{E}\left[\exp \left(u_{n} Y_{k, n}\right)-1\right]\right)^{2}\right| \\
& \quad \leq \frac{v_{n}^{2}}{2 a_{n}} \sum_{k=1}^{n}\left(\mathbb{E}\left[\exp \left(u_{n} Y_{k, n}\right)-1\right]\right)^{2} \\
& \leq c_{2} \frac{u^{2}}{2} h_{n}\|f\|_{\infty}^{2} \exp \left(\left|u_{n}\right|\|K\|_{\infty}\right)\left(\int_{\mathbb{R}}|\mathcal{K}(-z)| d z\right)^{2}
\end{aligned}
$$

The combination of (13) and (14) ensures that $\lim _{n \rightarrow \infty}\left|R_{n, x}^{(2)}(u)\right|=0$. Then, we obtain from (11), $\lim _{n \rightarrow \infty} \Lambda_{n, x}(u)=\Lambda_{x}^{M}(u)$. 
Second case: $\left(v_{n}\right) \equiv 1$. It follows from $(10)$, that

$$
\begin{aligned}
\Lambda_{n, x}(u)= & \frac{1}{a_{n}} \sum_{k=1}^{n} \mathbb{E}\left[\exp \left(u Y_{k, n}\right)-1\right]-\frac{1}{2 a_{n}} \sum_{k=1}^{n} \frac{1}{c_{k, n}^{2}}\left(\mathbb{E}\left[\exp \left(u Y_{k, n}\right)-1\right]\right)^{2} \\
& -u \mathbb{E}\left[Y_{1, n}\right]
\end{aligned}
$$

Moreover, using integration by parts, we get

$$
\Lambda_{n, x}(u)=u F(x) \int_{\mathbb{R}} K(z)(\exp (u \mathcal{K}(-z))-1) d z-R_{n, x}^{(3)}(u)+R_{n, x}^{(4)}(u)
$$

with

$$
\begin{aligned}
& R_{n, x}^{(3)}(u)=\frac{1}{2 a_{n}} \sum_{k=1}^{n} \frac{1}{c_{k, n}^{2}}\left(\mathbb{E}\left[\exp \left(u Y_{k, n}\right)-1\right]\right)^{2} \\
& R_{n, x}^{(4)}(u)=u \int_{\mathbb{R}} K(z)(\exp (u \mathcal{K}(-z))-1)\left[F\left(x+z h_{n}\right)-F(x)\right] d z .
\end{aligned}
$$

It follows from (14), that $\lim _{n \rightarrow \infty}\left|R_{n, x}^{(3)}(u)\right|=0$.

Since $\left|e^{t}-1\right| \leq|t| e^{|t|}$, we have

$$
\left|R_{n, x}^{(4)}(u)\right| \leq u^{2} e^{|u|\|\mathcal{K}\|_{\infty}} \int_{\mathbb{R}}|K(z)||\mathcal{K}(-z)|\left|F\left(x+z h_{n}\right)-F(x)\right| d z .
$$

Then, the dominated convergence theorem ensures that $\lim _{n \rightarrow \infty} R_{n, x}^{(4)}(u)=0$.

In the case $F$ is uniformly continuous, set $\varepsilon>0$ and let $M>0$ such that $2\|F\|_{\infty} \int_{\|z\| \leq M}|K(z)||\mathcal{K}(-z)| d z \leq \varepsilon / 2$. We need to prove that for $n$ sufficiently large

$$
\sup _{x \in \mathbb{R}} \int_{\|z\| \leq M}|K(z)||\mathcal{K}(-z)|\left|F\left(x+z h_{n}\right)-F(x)\right| d z \leq \varepsilon / 2
$$

which is a straightforward consequence of the uniform continuity of $F$.

Then, it follows from (15), that

$$
\begin{aligned}
\lim _{n \rightarrow \infty} \Lambda_{n, x}(u) & =u F(x) \int_{\mathbb{R}} K(z)(\exp (u \mathcal{K}(-z))-1) d z \\
& =F(x)(\exp (u)-1-u) \\
& =\Lambda_{x}^{L}(u)
\end{aligned}
$$

and thus Lemma 1 is proved. 


\subsection{Proof of Proposition 1}

To prove Proposition 1, we apply Lemma 1 and the following result (see Puhalskii, 1994).

Lemma 2. Let $\left(Z_{n}\right)$ be a sequence of real random variables, $\left(\nu_{n}\right)$ a positive sequence satisfying $\lim _{n \rightarrow \infty} \nu_{n}=+\infty$, and suppose that there exists some convex non-negative function $\Gamma$ defined on $\mathbb{R}$ such that

$$
\forall u \in \mathbb{R}, \lim _{n \rightarrow \infty} \frac{1}{\nu_{n}} \log \mathbb{E}\left[\exp \left(u \nu_{n} Z_{n}\right)\right]=\Gamma(u) .
$$

If the Legendre function $\Gamma^{*}$ of $\Gamma$ is a strictly convex function, then the sequence $\left(Z_{n}\right)$ satisfies a LDP of speed $\left(\nu_{n}\right)$ and good rate fonction $\Gamma^{*}$.

In our framework, when $v_{n} \equiv 1$, we take $Z_{n}=F_{n}(x)-\mathbb{E}\left(F_{n}(x)\right), \nu_{n}=n$ and $\Gamma=\Lambda_{x}^{L}$. In this case, the Legendre transform of $\Gamma=\Lambda_{x}^{L}$ is the rate function $I_{x}: t \rightarrow F(x) I\left(1+\frac{t}{F(x)}\right)$, since $\psi$ is strictly convex, then its Cramer transform $I$ is a good rate function on $\mathbb{R}$ (see Dembo and Zeitouni, 1998). Otherwise, when, $v_{n} \rightarrow \infty$, we take $Z_{n}=v_{n}\left(F_{n}(x)-\mathbb{E}\left(F_{n}(x)\right)\right), \nu_{n}=n / v_{n}^{2}$ and $\Gamma=\Lambda_{x}^{M} ; \Gamma^{*}$ is then the quadratic rate function $J_{x}$ defined in (3) and thus Proposition 1 follows.

\subsection{Proof of Proposition 2}

In order to prove Proposition 2, we first establish some lemmas.

Lemma 3. Let $\phi: \mathbb{R}^{+} \rightarrow \mathbb{R}$ be the function defined for $\delta>0$ as

$$
\phi(\delta)=\left\{\begin{array}{lll}
\left(\psi^{\prime}\right)^{-1}\left(1+\frac{\delta}{\|F\|_{U, \infty}}\right) & \text { when } v_{n} \equiv 1 \quad, \quad(L 1) \text { and }(L 2) \text { hold } \\
\frac{\delta}{\|F\|_{U, \infty}} & \text { when } v_{n} \rightarrow \infty \quad, \quad(M 1)-(M 4) \text { hold }
\end{array}\right.
$$

1. $\sup _{u \in \mathbb{R}}\left\{u \delta-\sup _{x \in U} \Lambda_{x}(u)\right\}$ equals $g_{U}(\delta)$ and is achieved for $u=\phi(\delta)>0$.

2. $\sup _{u \in \mathbb{R}}\left\{-u \delta-\sup _{x \in U} \Lambda_{x}(u)\right\}$ equals $g_{U}(\delta)$ and is achieved for $u=\phi(-\delta)<$ 0 .

Proof of Lemma 3 . We just prove the first part, the proof of the second part one being similar.

- First case $v_{n} \rightarrow 1$. Since $e^{t} \geq 1+t$, for all $t$, we have $\psi(u) \geq u$ and therefore,

$$
\begin{aligned}
u \delta-\sup _{x \in U} \Lambda_{x}(u) & =u \delta-\|F\|_{U, \infty}(\psi(u)-u) \\
& =\|F\|_{U, \infty}\left[u\left(1+\frac{\delta}{\|F\|_{U, \infty}}\right)-\psi(u)\right]
\end{aligned}
$$


The function $u \mapsto u \delta-\sup _{x \in U} \Lambda_{x}(u)$ has second derivative $-\|F\|_{U, \infty} \psi^{\prime \prime}(u)<0$ and thus it has a unique maximum achieved for

$$
u_{0}=\left(\psi^{\prime}\right)^{-1}\left(1+\frac{\delta}{\|F\|_{U, \infty}}\right)
$$

Now, since $\psi^{\prime}$ is increasing and since $\psi^{\prime}(0)=1$, we deduce that $u_{0}>0$.

- Second case $v_{n} \rightarrow \infty$. In this case, we have

$$
u \delta-\sup _{x \in U} \Lambda_{x}(u)=u \delta-\frac{u^{2}}{2}\|F\|_{U, \infty} .
$$

The function $u \mapsto u \delta-\sup _{x \in U} \Lambda_{x}(u)$ has second derivative $-\|F\|_{U, \infty}<0$ and thus it has a unique maximum achieved for

$$
u_{0}=\frac{\delta}{\|F\|_{U, \infty}}>0
$$

Lemma 4.

- In the case when $\left(v_{n}\right) \equiv 1$, let $(L 1)$ and $(L 2)$ hold;

- In the case when $v_{n} \rightarrow \infty$, let $(M 1)-(M 4)$ hold.

Then for any $\delta>0$,

$$
\begin{aligned}
\lim _{n \rightarrow \infty} \frac{v_{n}^{2}}{n} \log \sup _{x \in U} \mathbb{P}\left[v_{n} \Psi_{n}(x) \geq \delta\right] & =-g_{U}(\delta) \\
\lim _{n \rightarrow \infty} \frac{v_{n}^{2}}{n} \log \sup _{x \in U} \mathbb{P}\left[v_{n} \Psi_{n}(x) \leq-\delta\right] & =-g_{U}(-\delta) \\
\lim _{n \rightarrow \infty} \frac{v_{n}^{2}}{n} \log \sup _{x \in U} \mathbb{P}\left[v_{n}\left|\Psi_{n}(x)\right| \leq-\delta\right] & =-\tilde{g}_{U}(-\delta)
\end{aligned}
$$

Proof of Lemma 4. The proof of Lemma 4 is similar to the proof of Lemma 4 in Mokkadem et al. (2006).

Lemma 5. Let Assumptions $(U 1)-(U 3)$ hold and assume that either $\left(v_{n}\right) \equiv 1$ or (U4) holds.

1. If $U$ is a bounded set, then for any $\delta>0$, we have

$$
\lim _{n \rightarrow \infty} \frac{v_{n}^{2}}{n} \log \mathbb{P}\left[\sup _{x \in U} v_{n}\left|\Psi_{n}(x)\right|\right] \leq-\tilde{g}_{U}(\delta)
$$

2. If $U$ is an unbounded set, then, for any $b>0$ and $\delta>0$,

$$
\limsup _{n \rightarrow \infty} \frac{v_{n}^{2}}{n} \log \mathbb{P}\left[\sup _{x \in U,\|x\| \leq w_{n}} v_{n}\left|\Psi_{n}(x)\right|\right] \leq b-\tilde{g}_{U}(\delta)
$$

where $w_{n}=\exp \left(b \frac{n}{v_{n}^{2}}\right)$. 
Proof of Lemma 5. Set $\rho \in] 0, \delta\left[\right.$, let $\beta$ denote the Hölder order of $\mathcal{K}$, and $\|\mathcal{K}\|_{H}$ its corresponding Hölder norm. Set $w_{n}=\exp \left(b \frac{n}{v_{n}^{2}}\right)$ and

$$
R_{n}=\left(\frac{\rho}{2\|\mathcal{K}\|_{H} v_{n} h_{n}^{-\beta}}\right)^{\frac{1}{\beta}}
$$

We begin with the proof of the second part of Lemma 5. There exist $N^{\prime}(n)$ points of $\mathbb{R}, y_{1}^{(n)}, y_{2}^{(n)}, \ldots, y_{N^{\prime}(n)}^{(n)}$ such that the ball $\left\{x \in \mathbb{R} ;\|x\| \leq w_{n}\right\}$ can covered by the $N^{\prime}(n)$ balls $B_{i}^{(n)}=\left\{x \in \mathbb{R} ;\left\|x-y_{i}^{(n)}\right\| \leq R_{n}\right\}$ and such that $N^{\prime}(n) \leq 2\left(\frac{2 w_{n}}{R_{n}}\right)$. Considering only the $N(n)$ balls that intersect $\left\{x \in U ;\|x\| \leq w_{n}\right\}$, we can write

$$
\left\{x \in U ;\|x\| \leq w_{n}\right\} \subset \cup_{i=1}^{N(n)} B_{i}^{(n)} .
$$

For each $i \in\{1, \ldots, N(n)\}$, set $x_{i}^{(n)} \in B_{i}^{(n)} \cap U$. We then have:

$$
\begin{aligned}
\mathbb{P}\left[\sup _{x \in U,\|x\| \leq w_{n}} v_{n}\left|\Psi_{n}(x)\right| \geq \delta\right] & \leq \sum_{i=1}^{N(n)} \mathbb{P}\left[\sup _{x \in B_{i}^{(n)}} v_{n}\left|\Psi_{n}(x)\right| \geq \delta\right] \\
& \leq N(n) \max _{1 \leq i \leq N(n)} \mathbb{P}\left[\sup _{x \in B_{i}^{(n)}} v_{n}\left|\Psi_{n}(x)\right| \geq \delta\right]
\end{aligned}
$$

Now, for any $i \in\{1, \ldots, N(n)\}$ and any $x \in B_{i}^{(n)}$,

$$
\begin{aligned}
v_{n}\left|\Psi_{n}(x)\right| \leq & v_{n}\left|\Psi_{n}\left(x_{i}^{(n)}\right)\right| \\
& +\frac{v_{n}}{n} \sum_{k=1}^{n}\left|\mathcal{K}\left(\frac{x-X_{k}}{h_{n}}\right)-\mathcal{K}\left(\frac{x_{i}^{(n)}-X_{k}}{h_{n}}\right)\right| \\
& +\frac{v_{n}}{n} \sum_{k=1}^{n} \mathbb{E}\left|\mathcal{K}\left(\frac{x-X_{k}}{h_{n}}\right)-\mathcal{K}\left(\frac{x_{i}^{(n)}-X_{k}}{h_{n}}\right)\right| \\
\leq & v_{n}\left|\Psi_{n}\left(x_{i}^{(n)}\right)\right|+2 \frac{v_{n}}{n}\|\mathcal{K}\|_{H} \sum_{k=1}^{n}\left(\frac{\left\|x-x_{i}^{(n)}\right\|}{h_{n}}\right)^{\beta} \\
\leq & v_{n}\left|\left(x_{i}^{(n)}\right)\right|+2 v_{n}\|\mathcal{K}\|_{H} h_{n}^{-\beta} R_{n}^{\beta} \\
\leq & v_{n}\left|\Psi_{n}\left(x_{i}^{(n)}\right)\right|+\rho
\end{aligned}
$$

Hence, we deduce that

$$
\begin{aligned}
\mathbb{P}\left[\sup _{x \in U,\|x\| \leq w_{n}} v_{n}\left|\Psi_{n}(x)\right| \geq \delta\right] & \leq N(n) \max _{1 \leq i \leq N(n)} \mathbb{P}\left[v_{n}\left|\Psi_{n}\left(x_{i}^{(n)}\right)\right| \geq \delta-\rho\right] \\
& \leq N(n) \sup _{x \in U} \mathbb{P}\left[v_{n}\left|\Psi_{n}\left(x_{i}^{(n)}\right)\right| \geq \delta-\rho\right]
\end{aligned}
$$


Further, by definition of $N(n)$ and $w_{n}$, we have

$$
\log N(n) \leq \log N^{\prime}(n) \leq b \frac{n}{v_{n}^{2}}+2 \log 2-\log R_{n}
$$

and

$$
\frac{v_{n}^{2}}{n} \log R_{n}=\frac{1}{\beta} \frac{v_{n}^{2}}{n}\left[\log \rho-\log \left(2\|\mathcal{K}\|_{H}\right)-\log v_{n}+\beta \log h_{n}\right]
$$

Then, in view of $(U 3)$, we have

$$
\limsup _{n \rightarrow \infty} \frac{v_{n}^{2}}{n} \log N(n) \leq b
$$

The application of Lemma 4 then yields

$$
\begin{aligned}
\limsup _{n \rightarrow \infty} \frac{v_{n}^{2}}{n} \log \mathbb{P}\left[\sup _{x \in U,\|x\| \leq w_{n}} v_{n}\left|\Psi_{n}(x)\right| \geq \delta\right] & \leq \limsup _{n \rightarrow \infty} \frac{v_{n}^{2}}{n} \log N(n)-\tilde{g}_{U}(\delta-\rho) \\
& \leq b-\tilde{g}_{U}(\delta-\rho) .
\end{aligned}
$$

Since the inequality holds for any $\rho \in] 0, \delta[$, part 2 of Lemma 5 thus follows from the continuity of $\tilde{g}_{U}$.

Let us now consider part 1 of Lemma 5. This part is proved by following the same steps as for part 2, except that the number $N(n)$ of balls covering $U$ is at most the integer part of $\left(\Delta / R_{n}\right)$, where $\Delta$ denotes the diameter of $\bar{U}$. Relation (16) then becomes

$$
\limsup _{n \rightarrow \infty} \frac{v_{n}^{2}}{n} \log R_{n} \leq 0
$$

and Lemma 5 is proved.

Lemma 6. Let $(U 1) i),(M 2)$ and $(U 6) i)$ hold. Assume that either $\left(v_{n}\right) \equiv 1$ or $(U 3)$ and (U6) $i i$ ) hold. Moreover assume that $F$ is continuous. For any $b>0$ if we set $w_{n}=\exp \left(b \frac{n}{v_{n}^{2}}\right)$ then, for any $\rho>0$, we have, for $n$ large enough,

$$
\sup _{x \in U,\|x\| \geq w_{n}} \frac{v_{n}}{n} \sum_{k=1}^{n}\left|\mathbb{E}\left[\mathcal{K}\left(\frac{x-X_{k}}{h_{n}}\right)\right]\right| \leq \rho
$$

Proof of Lemma 6. We have

$$
\frac{v_{n}}{n} \sum_{k=1}^{n} \mathbb{E}\left[\mathcal{K}\left(\frac{x-X_{k}}{h_{n}}\right)\right]=v_{n} \int_{\mathbb{R}} K(z) F\left(x+z h_{n}\right) d z .
$$


Set $\rho>0$. In the case $\left(v_{n}\right) \equiv 1$, we set $M$ such that $\|F\|_{\infty} \int_{\|z\|>M}|K(z)| d z \leq \rho / 2$; it follows that

$$
\begin{aligned}
& \frac{v_{n}}{n} \sum_{k=1}^{n}\left|\mathbb{E}\left[\mathcal{K}\left(\frac{x-X_{k}}{h_{n}}\right)\right]\right| \\
& \quad \leq \frac{\rho}{2}+F(x) \int_{\|z\| \leq M}|K(z)| d z+\int_{\|z\|>M}|K(z)|\left|F\left(x+z h_{n}\right)-F(x)\right| d z .
\end{aligned}
$$

Lemma 6 then follows from the fact that $F$ fulfills $(U 6)$ ii). As matter of fact, this conditions implies that $\lim _{\|x\| \rightarrow \infty, x \in \bar{U}} F(x)=0$ and that the third term in the right-hand-side of the previous inequality goes to 0 as $n \rightarrow \infty$ (by the dominated convergence).

Let us now assume that $\lim _{n \rightarrow \infty} v_{n}=\infty$; relation (17) can be rewritten as

$$
\begin{aligned}
\frac{v_{n}}{n} \sum_{k=1}^{n} \mathbb{E}\left[\mathcal{K}\left(\frac{x-X_{k}}{h_{n}}\right)\right]= & v_{n} \int_{\|z\| \leq w_{n} / 2} K(z) F\left(x+z h_{n}\right) d z \\
& +v_{n} \int_{\|z\| \geq w_{n} / 2} K(z) F\left(x+z h_{n}\right) d z
\end{aligned}
$$

First, since $\|x\| \geq w_{n}$ and $\|z\| \leq w_{n} / 2$, we have

$$
\begin{aligned}
\left\|x+z h_{n}\right\| & \geq w_{n}\left(1-h_{n} / 2\right) \\
& \geq w_{n} / 2 \text { for } n \text { large enough. }
\end{aligned}
$$

Moreover, in view of assumptions $(U 3)$, for all $\xi>0$,

$$
\lim _{n \rightarrow \infty} \frac{v_{n}}{w_{n}^{\xi}}=\lim _{n \rightarrow \infty} \exp \left\{-b \xi \frac{n}{v_{n}^{2}}\left(1-\frac{1}{b \xi} \frac{v_{n}^{2} \log v_{n}}{n}\right)\right\}=0
$$

Set $M_{f}=\sup _{x \in \mathbb{R}}\|x\|^{\eta} F(x)$. Assumption (U6)ii) and equation (18) implie that, for $n$ sufficiently large,

$$
\begin{aligned}
& \sup _{\|x\| \geq w_{n}} v_{n} \int_{\|z\| \leq w_{n} / 2}\left|K(z) F\left(x+z h_{n}\right)\right| d z \\
& \quad \leq M_{f} \sup _{\|x\| \geq w_{n}} v_{n} \int_{\|z\| \leq w_{n} / 2}|K(z)|\left\|x+z h_{n}\right\|^{-\eta} d z \\
& \quad \leq 2^{\eta} M_{f} \frac{v_{n}}{w_{n}^{\eta}} \int_{\mathbb{R}}|K(z)| d z \\
& \quad \leq \frac{\rho}{2}
\end{aligned}
$$


Moreover, in view of (U3), (U6) $i)$ and (18), for $n$ sufficiently large,

$$
\begin{aligned}
& \sup _{\|x\| \geq w_{n}} v_{n} \int_{\|z\|>w_{n} / 2}\left|K(z) F\left(x+z h_{n}\right)\right| d z \\
& \quad \leq 2^{\zeta} M_{f} \frac{v_{n}}{w_{n}^{\zeta}} \int_{\|z\|>w_{n} / 2}\|z\|^{\zeta}|K(z)| d z \\
& \quad \leq \frac{\rho}{2}
\end{aligned}
$$

This concludes the proof of Lemma 6 . Since $K$ is a bounded function that vanishes at infinity, we have $\lim _{\|x\| \rightarrow \infty}\left|\Psi_{n}(x)\right|=0$ for every $n \geq 1$. Moreover, since $K$ is assumed to be continuous, $\Psi_{n}$ is continuous, and this ensures the existence of a random variable $s_{n}$ such that

$$
\left|\Psi_{n}\left(s_{n}\right)\right|=\sup _{x \in U}\left|\Psi_{n}(x)\right|
$$

\section{Lemma 7.}

Let Assumptions $(U 1)-(U 3),(U 4) i i)$ and $(U 5)$ hold. Suppose either $\left(v_{n}\right) \equiv 1$ or (H6) hold. For any $b>0$, set $w_{n}=\exp \left(b \frac{n}{v_{n}^{2}}\right)$; for any $\delta>0$, we have

$$
\limsup _{n \rightarrow \infty} \frac{v_{n}^{2}}{n} \log \mathbb{P}\left[\left\|s_{n}\right\| \geq w_{n} \quad \text { and } \quad\left|\Psi_{n}\left(s_{n}\right)\right| \geq \delta\right] \leq-b \beta
$$

Proof of Lemma 7. We first note that $s_{n} \in \bar{U}$ and therefore

$$
\begin{gathered}
\left\|s_{n}\right\| \geq w_{n} \quad \text { and } \quad v_{n}\left|\Psi_{n}\left(s_{n}\right)\right| \geq \delta \\
\Rightarrow\left\|s_{n}\right\| \geq w_{n} \quad \text { and } \quad \frac{v_{n}}{n}\left|\sum_{k=1}^{n} \mathcal{K}\left(\frac{s_{n}-X_{k}}{h_{n}}\right)\right|+\frac{v_{n}}{n} \mathbb{E}\left|\sum_{k=1}^{n} \mathcal{K}\left(\frac{s_{n}-X_{k}}{h_{n}}\right)\right| \geq \delta \\
\Rightarrow\left\|s_{n}\right\| \geq w_{n} \text { and } \frac{v_{n}}{n} \sum_{k=1}^{n}\left|\mathcal{K}\left(\frac{s_{n}-X_{k}}{h_{n}}\right)\right| \geq \delta \\
-\sup _{\|x\| \geq w_{n}, x \in \bar{U}} \frac{v_{n}}{n} \sum_{k=1}^{n} \mathbb{E}\left|\mathcal{K}\left(\frac{s_{n}-X_{k}}{h_{n}}\right)\right|
\end{gathered}
$$

Set $\rho \in] 0, \delta[$; the application of Lemma 6 ensures that, for $n$ large enough,

$$
\begin{aligned}
&\left\|s_{n}\right\| \geq w_{n} \quad \text { and } \quad v_{n}\left|\Psi_{n}\left(s_{n}\right)\right| \geq \delta \\
& \Rightarrow\left\|s_{n}\right\| \geq w_{n} \quad \text { and } \quad \frac{v_{n}}{n}\left|\sum_{k=1}^{n} \mathcal{K}\left(\frac{s_{n}-X_{k}}{h_{n}}\right)\right| \geq \delta-\rho .
\end{aligned}
$$


Set $\kappa=\sup _{x \in \mathbb{R}}\|x\|^{\gamma}|\mathcal{K}(x)|$ (see Assumption (U5)). We obtain, for $n$ sufficiently large,

$$
\begin{aligned}
&\left\|s_{n}\right\| \geq w_{n} \quad \text { and } \quad v_{n}\left|\Psi_{n}\left(s_{n}\right)\right| \geq \delta \\
& \Rightarrow\left\|s_{n}\right\| \geq w_{n} \quad \text { and } \quad \exists k \in\{1, \ldots, n\} \quad \text { such that } \quad v_{n}\left|\mathcal{K}\left(\frac{s_{n}-X_{k}}{h_{n}}\right)\right| \geq \delta-\rho \\
& \Rightarrow\left\|s_{n}\right\| \geq w_{n} \quad \text { and } \quad \exists k \in\{1, \ldots, n\} \quad \text { such that } \quad \kappa h_{n}^{\gamma} \geq v_{n}^{-1}\left\|s_{n}-X_{k}\right\|^{\gamma}(\delta-\rho) \\
& \Rightarrow\left\|s_{n}\right\| \geq w_{n} \quad \text { and } \quad \exists k \in\{1, \ldots, n\} \quad \text { such that } \quad\left\|s_{n}\right\|-\left\|X_{k}\right\| \mid \leq\left[\frac{\kappa v_{n} h_{n}^{\gamma}}{\delta-\rho}\right]^{\frac{1}{\gamma}} \\
& \Rightarrow\left\|s_{n}\right\| \geq w_{n} \text { and } \quad \exists k \in\{1, \ldots, n\} \quad \text { such that }\left\|X_{k}\right\| \leq\left\|s_{n}\right\|-\left[\frac{\kappa v_{n} h_{n}^{\gamma}}{\delta-\rho}\right]^{\frac{1}{\gamma}} \\
& \Rightarrow\left\|s_{n}\right\| \geq w_{n} \text { and } \quad \exists k \in\{1, \ldots, n\} \quad \text { such that } \quad\left\|X_{k}\right\| \leq w_{n}\left(1-u_{n, k}\right) \text { with } \\
& u_{n, k}=w_{n}^{-1} v_{n}^{\frac{1}{\gamma}} h_{n}\left(\frac{\kappa}{\delta-\rho}\right)^{\frac{1}{\gamma}} .
\end{aligned}
$$

Moreover, we can write $u_{n, k}$ as

$$
u_{n, k}=\exp \left(-b \frac{n}{v_{n}^{2}}\left[1-\frac{v_{n}^{2} \log v_{n}}{n} \frac{1}{b \gamma}-\frac{v_{n}^{2} \log \left(h_{n}\right)}{n} \frac{1}{b}\right]\right)\left(\frac{\kappa}{\delta-\rho}\right)^{\frac{1}{\gamma}}
$$

and assumption (U3) ensure that $\lim _{n \rightarrow \infty} u_{n, k}=0$, it then follows that $1-u_{n, k}>0$ for $n$ sufficiently large; therefore we can deduce that (see Assumption $(U 4) i)$ ):

$$
\begin{aligned}
\mathbb{P}\left[\left\|s_{n}\right\| \geq w_{n} \quad \text { and } \quad v_{n}\left|\Psi_{n}\left(s_{n}\right)\right| \geq \delta\right] & \leq \sum_{i=1}^{n} \mathbb{P}\left[\left\|X_{k}\right\|^{\beta} \geq w_{n}^{\beta}\left(1-u_{n, k}\right)^{\beta}\right] \\
& \leq \sum_{i=1}^{n} \mathbb{E}\left(\left\|X_{k}\right\|^{\beta}\right) w_{n}^{-\beta}\left(1-u_{n, k}\right)^{-\beta} \\
& \leq n \mathbb{E}\left(\left\|X_{1}\right\|^{\beta}\right) w_{n}^{-\beta} \max _{1 \leq k \leq n}\left(1-u_{n, k}\right)^{-\beta} .
\end{aligned}
$$

Consequently,

$$
\begin{aligned}
& \frac{v_{n}^{2}}{n} \log \mathbb{P}\left[\left\|s_{n}\right\| \geq w_{n} \quad \text { and } \quad v_{n}\left|\Psi_{n}\left(s_{n}\right)\right| \geq \delta\right] \\
& \quad \leq \frac{v_{n}^{2}}{n}\left[\log n+\log \mathbb{E}\left(\left\|X_{1}\right\|^{\beta}\right)-b \beta n v_{n}^{2}-\beta \log \max _{1 \leq k \leq n}\left(1-u_{n, k}\right)\right]
\end{aligned}
$$

and, thanks to assumptions $(U 3)$, it follows that

$$
\limsup _{n \rightarrow \infty} \frac{v_{n}^{2}}{n} \log \mathbb{P}\left[\left\|s_{n}\right\| \geq w_{n} \quad \text { and } \quad v_{n}\left|\Psi_{n}\left(s_{n}\right)\right| \geq \delta\right] \leq-b \beta,
$$

which concludes the proof of Lemma 7. 


\subsection{Proof of Proposition 2}

Let us at first note that the lower bound

$$
\liminf _{n \rightarrow \infty} \frac{v_{n}^{2}}{n} \log \mathbb{P}\left[\sup _{x \in U} v_{n}\left|\Psi_{n}(x)\right| \geq \delta\right] \geq-\tilde{g}_{U}(\delta)
$$

follows from the application of Proposition 1 at a point $x_{0} \in \bar{U}$ such that $F\left(x_{0}\right)=$ $\|F\|_{U, \infty}$.

In the case $U$ is bounded, Proposition 2 is thus a straightforward consequence of (20) and the first part of Lemma 5. Let us now consider the case $U$ is unbounded.

Set $\delta>0$ and, for any $b>0$ set $w_{n}=\exp \left(b \frac{n}{v_{n}^{2}}\right)$. Since, by definition of $s_{n}$,

$$
\begin{aligned}
& \mathbb{P}\left[\sup _{x \in U} v_{n}\left|\Psi_{n}(x)\right| \geq \delta\right] \\
& \quad \leq \mathbb{P}\left[\sup _{x \in U,\|x\| \leq w_{n}} v_{n}\left|\Psi_{n}(x)\right| \geq \delta\right]+\mathbb{P}\left[\left\|s_{n}\right\| \geq w_{n} \text { and } v_{n}\left|\Psi_{n}(x)\right| \geq \delta\right],
\end{aligned}
$$

it follows from Lemmas 5 and 7 that

$$
\limsup _{n \rightarrow \infty} \frac{v_{n}^{2}}{n} \log \mathbb{P}\left[\sup _{x \in U} v_{n}\left|\Psi_{n}(x)\right| \geq \delta\right] \leq \max \left\{-b \beta ; b-\tilde{g}_{U}(\delta)\right\}
$$

and consequently

$$
\limsup _{n \rightarrow \infty} \frac{v_{n}^{2}}{n} \log \mathbb{P}\left[\sup _{x \in U} v_{n}\left|\Psi_{n}(x)\right| \geq \delta\right] \leq \inf _{b>0} \max \left\{-b \beta ; b-\tilde{g}_{U}(\delta)\right\} .
$$

Since the infimum in the right-hand-side of the previous bound is achieved for $b=$ $\tilde{g}_{U}(\delta) /(\beta+1)$ and equals $-\beta \tilde{g}_{U}(\delta) /(\beta+1)$, we obtain the upper bound

$$
\limsup _{n \rightarrow \infty} \frac{v_{n}^{2}}{n} \log \mathbb{P}\left[\sup _{x \in U} v_{n}\left|\Psi_{n}(x)\right| \geq \delta\right] \leq-\frac{\beta}{\beta+1} \tilde{g}_{U}(\delta)
$$

which concludes the proof of Proposition 2.

\subsection{Proof of Proposition 3}

It follows from (1), that

$$
\begin{aligned}
\mathbb{E}\left[F_{n}(x)\right] & =\int_{\mathbb{R}} \mathcal{K}\left(\frac{x-y}{h_{n}}\right) f(y) d y \\
& =\int_{\mathbb{R}} K(z) F\left(x+z h_{n}\right) d z \\
& =F(x)+\frac{1}{2} h_{n}^{2} F^{(2)}(x) \int_{\mathbb{R}} z^{2} K(z) d z+\eta(x)
\end{aligned}
$$


with

$$
\eta(x)=\int_{\mathbb{R}}\left[F\left(x+z h_{n}\right)-F(x)-z h_{n} F^{\prime}(x)-\frac{1}{2} z^{2} h_{n}^{2} F^{(2)}(x)\right] K(z) d z
$$

Since $F$ is continuous, we have $\lim _{n \rightarrow \infty}\left|F\left(x+z h_{n}\right)-F(x)-z h_{n} F^{\prime}(x)-\frac{1}{2} z^{2} h_{n}^{2} F^{(2)}(x)\right|=$ 0 , and thus by the dominated convergence theorem, we have $\lim _{n \rightarrow \infty} \eta(x)=0$, and thus Part 1 of Proposition 3 is completed. Since $\sup _{x \in \mathbb{R}}\left\|F^{(2)}(x)\right\|<+\infty$, Part 2 follows.

\section{References}

A. Azzalini, A note on the estimation of a distribution function and quantiles by a kernel method, Biometrika, 68 (1981), 326-328.

R. Bojanic and E. Seneta, A unified theory of regularly varying sequences, Math. Z, 134 (1973), 91-106.

A. Dembo and O. Zeitouni, Large deviations techniques and applications. Springer, Application of mathematics, New-York, 1998

M. Falk, Relative efficiency and deficiency of kernel type estimator of smooth distribution functions, Stat. Neerl, 37 (1983), 73-83.

J. Galambos and E. Seneta, Regularly varying sequences, Amer. Math. Soc, 41 (1973), 110-116.

F. Gao, Moderate deviations and large deviations for kernel density estimators, $J$. Theoret. Probab, 16 (2003), 401-418.

P.D. Hill, Kernel estimation of a distribution function, Commun. Stat-Theor. M, 14 (1985), 605-620.

D. Louani, Large deviations limit theorems for the kernel density estimator, Scand. J. Statist, 25 (1998), 243-253.

A. Mokkadem \& M. Pelletier and J. Worms, Large and moderate deviations principles for kernel estimation of a multivariate density and its partial derivatives, Austral. J. Statist, 4 (2005), 489-502.

A. Mokkadem, M. Pelletier and B. Thiam, Large and moderate deviations principles for recursive kernel estimation of a multivariate density and its partial derivatives, Serdica Math. J, 32 (2006), 323-354.

E.A. Nadaraya, Some new estimates for distribution functions. Theory Prob. Appl, 9 (1964), 497-500. 
E. Parzen, On estimate of a probability density and mode, Ann. Math. Statist, 33 (1962), 1065-1076.

A.A. Puhalskii, The method of stochastic exponentials for large deviations, Stochastic Process. Appl, 54 (1994), 45-70.

R.D. Reiss, Nonparametric estimation of smooth distribution function, Scand. J. Statist, 8 (1981), 116-119.

M. Rosenblatt, Remarks on some nonparametric estimate of a density function, Ann. Math. Statist, 27 (1956), 832-837.

J. Worms, Moderate and large deviations of some dependent variables, Part II: Some kernel estimators, Math. Methods Statist, 10 (2001), 161-193.

Received: May 1, 2014 